\title{
结构不对称三芳胺类化合物的合成、表征及性质研究
}

\author{
李英俊* 赵 楠李丽娜李春燕孙淑琴周晓霞
}

(辽宁师范大学化学化工学院 大连 116029)

\begin{abstract}
摘要 以二芳胺和 3,5-二甲基碘苯为原料, 利用乌尔曼(Ullmann)反应, 合成出了 4 个结构不对称三芳胺衍生物 $\mathbf{1} \sim \mathbf{4}$, 除了化合物 1 之外均为新化合物. 利用 IR, ${ }^{1} \mathrm{H}$ NMR, ${ }^{13} \mathrm{C}$ NMR, HRMS 和元素分析对其结构进行了表征. 考察了它们的 光学、电化学和热稳定性质. 实验结果表明, 所合成的 4 个目标化合物在氯仿稀溶液中发出绿色苂光, 具有良好的电化 学和热稳定性. 所合成的目标化合物是潜在的空穴传输材料及发绿光的材料.
\end{abstract}

关键词 三芳胺; 空穴传输材料; 合成; 性质研究

\section{Synthesis, Characterization and Properties of Some Novel Structural Asymmetric Triarylamines}

\author{
Li, Yingjun* Zhao, Nan Li, Lina Li, Chunyan Sun, Shuqin Zhou, Xiaoxia
}

(College of Chemistry and Chemical Engineering, Liaoning Normal University, Dalian 116029)

\begin{abstract}
Four structural asymmetric triarylamines were synthesized by diarylamines and 3,5-dimethyl-iodobenzene as the starting materials using Ullmann reaction. They are new compounds except the compound $\mathbf{1}$. The structures of the target compounds were characterized by IR, ${ }^{1} \mathrm{H}$ NMR, ${ }^{13} \mathrm{C}$ NMR, HRMS and elemental analysis. The optical, electrochemical, and thermal properties were examined. The results indicated that the synthesized compounds emitted green fluorescence in chloroform, and exhibited a good electrochemical and thermal stability. The synthesized triarylamines are potential hole-transporting materials and green-light-emitting materials.
\end{abstract}

Keywords triarylamine; hole-transporting material; synthesis; property research

空穴传输分子已被用于有机电子设备中，如有机发 光二极管(OLEDs)、场效应晶体管 (OFETs)和光电电池, 所以已被广泛研究 ${ }^{[1]}$. 例如化合物 TPA(三苯胺), TPD $\left[N, N^{\prime} \text {-二苯基- } N, N^{\prime} \text {-二(3-甲苯基)-1, } 1^{\prime} \text { - 联苯-4, } 4^{\prime} \text {-二胺 }\right]^{[2]}$ 和 NPB $\left[N, N^{\prime}\right.$-二苯基- $N, N^{\prime}$-二(1-菜基)-1, $1^{\prime}$-联苯-4, $4^{\prime}$-二 胺 ${ }^{[3]}$ 已被应用于有机发光二极管(OLEDs)领域. 空穴传 输材料(HTMs) 是有机电致发光器件的重要组成部分 ${ }^{[4]}$, 三芳胺类化合物由于其具有高的空穴迁移率和可逆的 氧化还原性能 ${ }^{[5,6]}$ 已成为目前最常用小分子空穴传输材 料之一, 并且已引起人们极大的兴趣. 目前的研究结果 表明, 三苯胺类化合物具有较好的给电子性、较低的离 子化电位、较高的空穴迁移率(一般在 $10^{-3} \sim 10^{-5}$ $\mathrm{cm}^{2} / \mathrm{Vs}$ )、较好的溶解性与无定形成膜性、较强的苂光性 能与光稳定性. 但三苯胺(TPA)其相对分子量小、溶解度 差、易析晶, 作为空穴传输材料时, 有易造成材料发脆
等一系列缺点. 此外, 根据文献[7]还可知，在三苯胺的 苯环上吸电子取代基的存在, 将强烈地阻碍三芳胺作为 HTMs 的性能. 因此, 我们设计在 TPA 的苯环上引入供 电子取代基，以降低分子的对称性，从而改善材料性质， 并利用 Ullmann 反应，合成出一系列新的结构不对称三 芳胺类化合物, 期望新化合物具有较好的作为 HTMs 的 性能和特性. 目标化合物 $\mathbf{1} \sim \mathbf{4}$ 的合成路线见 Scheme 1.

\section{1 实验部分}

\section{1 仪器和试剂}

仪器：X-5 显微熔点测定仪(温度计未经校正); WGH-30 型双光束红外分光光度计，液膜或 $\mathrm{KBr}$ 压片; 德国 BRUKER $500 \mathrm{MHz}$ 核磁共振仪, $\mathrm{CDCl}_{3}$ 为溶剂, TMS 为内标; Waters 公司生产的 GCT CA156 型质谱仪; 德国 Elementar 公司 Vario EL 型元素分析仪; 美国 TA

\footnotetext{
* E-mail: chemlab.lnnu@163.com

Received May 12, 2011; revised October 9, 2011; accepted January 6, 2012.

Project supported by the Natural Science Foundation of Liaoning Province (No. 20102126).

辽宁省自然科学基金(No. 20102126)资助项目.
} 




Scheme 1

公司 SDT Q600 DSC/TGA 同步热分析仪; 日本岛津 UV-240 紫外-可见分光光度计; HITACHI F-4600 荧光光 谱仪; LK2005 型电化学工作站. 所用试剂均为分析纯试 剂.

\section{2 目标化合物的合成}

在氮气保护下向配有分水器、电动搅拌器、回流冷 凝器的 $100 \mathrm{~mL}$ 干燥的三口瓶中, 依次加入 $6.22 \mathrm{mmol}$ 未取代(取代的)二苯胺, $3.66 \mathrm{mmol} 3,5$-二甲基碘苯,

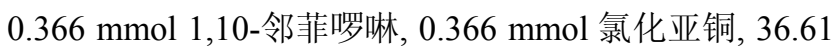
$\mathrm{mmol}$ 片状 $\mathrm{KOH}$ 和 $20 \mathrm{~mL}$ 甲苯, 加热回流反应 $20 \mathrm{~h}$. 反 应结束后, 向反应瓶中加入 $20 \mathrm{~mL}$ 甲苯和 $20 \mathrm{~mL}$ 水摚拌 $30 \mathrm{~min}$, 然后将反应液进行抽虑, 将滤液转移至分液漏 斗中, 分出有机相, 水洗有机相至 $\mathrm{pH}=7$, 用无水 $\mathrm{MgSO}_{4}$ 干燥, 活性炭脱色后, 减压蒸出溶剂. 将所得粗 产物经硅胶柱层析分离提纯后即得到纯品目标化合物 $1 \sim 4$.

目标化合物 2 4 为新化合物, 化合物 1 虽然为已知 物, 但有关其性质的研究尚未见报道. 目标化合物 $\mathbf{1} \sim \mathbf{4}$ 和 TPA 的热、光、电化学性质的物理学测定结果见表 1.

DMTPA [ $N$-(3,5-二甲基苯基)二苯胺, 1]: 白色针状 晶体, 产率 $55.3 \%, R_{\mathrm{f}} 0.27$ [石油醚 $\left(60 \sim 90{ }^{\circ} \mathrm{C}\right)$ ]. m.p. $134.7 \sim 135.2{ }^{\circ} \mathrm{C}$ (文献值 ${ }^{88]} 136.5{ }^{\circ} \mathrm{C}$ ); ${ }^{1} \mathrm{H}$ NMR $\left(\mathrm{CDCl}_{3}\right.$,
$500 \mathrm{MHz}) \delta: 7.22(\mathrm{t}, J=8.0 \mathrm{~Hz}, 4 \mathrm{H}, \mathrm{ArH}), 7.06(\mathrm{dd}, J=$ 8.6, $1.0 \mathrm{~Hz}, 4 \mathrm{H}, \mathrm{ArH}), 6.98$ (tt, $J=7.3,1.1 \mathrm{~Hz}, 2 \mathrm{H}, \mathrm{ArH})$, $6.71(\mathrm{~s}, 2 \mathrm{H}, \mathrm{ArH}), 6.67(\mathrm{~s}, 1 \mathrm{H}, \mathrm{ArH}), 2.21(\mathrm{~s}, 6 \mathrm{H}, 2 \times$ $\mathrm{CH}_{3}$ ); IR (KBr) v: 3032, 2916, 2856, 1590, 1494, 1380, $1308 \mathrm{~cm}^{-1}$.

$m$ - $\mathrm{CH}_{3}$-DMTPA [ $\mathrm{N}$-(3-甲基苯基)- $\mathrm{N}$-(3,5-二甲基苯 基)苯胺, 2]: 白色固体, 产率 $33.2 \%, R_{\mathrm{f}} 0.74$ [ $V$ (石油醚, $\left.60 \sim 90{ }^{\circ} \mathrm{C}\right): V($ EtOAc $\left.)=25: 1\right]$. m.p. $78.5 \sim 80.4{ }^{\circ} \mathrm{C}$; ${ }^{1} \mathrm{H}$ NMR $\left(\mathrm{CDCl}_{3}, 500 \mathrm{MHz}\right) \delta: 7.21(\mathrm{t}, J=7.9 \mathrm{~Hz}, 2 \mathrm{H}$, ArH), 7.12 (t, $J=7.8 \mathrm{~Hz}, 1 \mathrm{H}, \mathrm{ArH}), 7.04$ (dd, $J=8.1,0.9$ $\mathrm{Hz}, 2 \mathrm{H}, \mathrm{ArH}), 6.97$ (t, J=7.3 Hz, 1H, ArH), 6.89 (s, 1H, ArH), 6.86 (dd, $J=7.8,2.2 \mathrm{~Hz}, 1 \mathrm{H}, \mathrm{ArH}), 6.81$ (d, $J=7.6$ $\mathrm{Hz}, 1 \mathrm{H}, \mathrm{ArH}), 6.70$ (s, 2H, ArH), 6.67 (s, 1H, ArH), 2.25 (s, 3H, $\mathrm{CH}_{3}$ ), 2.21 (s, $\left.6 \mathrm{H}, 2 \times \mathrm{CH}_{3}\right) ;{ }^{13} \mathrm{C}$ NMR (DMSO- $d_{6}$, $125 \mathrm{MHz}) \delta$ : 147.5, 147.4, 147.2, 138.6, 138.4, 129.3, $129.2,124.7,124.2,123.5,123.3,122.3,121.8,121.0$, 20.9, 20.8; IR (film) $v: 3032,2916,2868,1590,1492$, $1378,1318 \mathrm{~cm}^{-1}$. Anal. calcd for $\mathrm{C}_{21} \mathrm{H}_{21} \mathrm{~N}: \mathrm{C} 87.76, \mathrm{H}$ 7.36, N 4.87; found C 87.92, H 7.15, N 4.99. HRMS calcd for $\mathrm{C}_{21} \mathrm{H}_{21} \mathrm{~N}$ 287.1674, found 287.1674.

$p$ - $\mathrm{CH}_{3}$-DMTPA［ $\mathrm{N}$-(4-甲基苯基)- $\mathrm{N}$-(3,5-二甲基苯 基)苯胺, 3]：白色固体，产率 $60.7 \%, R_{\mathrm{f}} 0.77$ [ $\mathrm{V}$ (石油醚, $\left.60 \sim 90{ }^{\circ} \mathrm{C}\right): V($ EtOAc $\left.)=25: 1\right]$. m.p. $96 \sim 97.5{ }^{\circ} \mathrm{C} ;{ }^{1} \mathrm{H}$ NMR $\left(\mathrm{CDCl}_{3}, 500 \mathrm{MHz}\right) \delta: 7.20(\mathrm{t}, J=7.8 \mathrm{~Hz}, 2 \mathrm{H}, \mathrm{ArH})$, $7.04(\mathrm{~d}, J=8.4 \mathrm{~Hz}, 2 \mathrm{H}, \mathrm{ArH}), 7.03(\mathrm{~d}, J=8.7 \mathrm{~Hz}, 2 \mathrm{H}$, ArH), $6.98(\mathrm{~d}, J=8.3 \mathrm{~Hz}, 2 \mathrm{H}, \operatorname{ArH}), 6.94(\mathrm{t}, \quad J=7.3 \mathrm{~Hz}$, $1 \mathrm{H}, \mathrm{ArH}), 6.69(\mathrm{~s}, 2 \mathrm{H}, \mathrm{ArH}), 6.64(\mathrm{~s}, 1 \mathrm{H}, \mathrm{ArH}), 2.31(\mathrm{~s}$, $3 \mathrm{H}, \mathrm{CH}_{3}$ ), $2.20\left(\mathrm{~s}, 6 \mathrm{H}, 2 \times \mathrm{CH}_{3}\right) ;{ }^{13} \mathrm{C}$ NMR (DMSO- $d_{6}, 125$ MHz) $\delta$ : 147.6, 147.4, 144.8, 138.4, 132.2, 129.9, 129.2, 124.4, 124.3, 122.8, 122.0, 121.2, 20.9, 20.3; IR (KBr) v: 3036, 2916, 2860, 1590, 1506, 1488, 1472, 1378, 1300 $\mathrm{cm}^{-1}$. Anal. calcd for $\mathrm{C}_{21} \mathrm{H}_{21} \mathrm{~N}$ : C 87.76, H 7.36, N 4.87; found $\mathrm{C} 87.55, \mathrm{H}$ 7.62, N 5.12. HRMS calcd for $\mathrm{C}_{21} \mathrm{H}_{21} \mathrm{~N}$ 287.1674, found 287.1675.

$m$ - $\mathrm{CH}_{3} \mathrm{O}$-DMTPADMTPA [ $\mathrm{N}$-(3-甲氧基苯基)- $\mathrm{N}$ (3,5-二甲基苯基)苯胺]，5)：浅棕色粘稠液体，产率

表 1 目标化合物 1 4 的物理学测定结果

Table 1 Summary of the physical measurements of target compounds $\mathbf{1} \sim \mathbf{4}$

\begin{tabular}{clccccccc}
\hline Compd. & \multicolumn{1}{c}{$T_{\mathrm{m}}{ }^{a} /{ }^{\circ} \mathrm{C}$} & $T_{5 \mathrm{~d}}{ }^{a} /{ }^{\circ} \mathrm{C}$ & $T_{\mathrm{g}}{ }^{a} /{ }^{\circ} \mathrm{C}$ & $\lambda_{\max }{ }^{b} / \mathrm{nm}$ & $\lambda_{\mathrm{em}}{ }^{c} / \mathrm{nm}$ & $E_{\mathrm{g}}{ }^{d} / \mathrm{eV}$ & $E_{\mathrm{HOMO}} / \mathrm{eV}$ & $E_{\mathrm{LUMO}} / \mathrm{eV}$ \\
\hline TPA & $127.2(125 \sim 127)^{[9]}$ & 183.0 & 55 & 296 & 501 & 3.48 & -5.508 & -2.028 \\
$\mathbf{1}$ & $135.5(136.5)^{[8]}$ & 198.7 & 55 & 298 & 502 & 3.43 & -5.364 & -1.934 \\
$\mathbf{2}$ & 84.6 & 191.9 & 55 & 299 & 502 & 3.38 & -5.374 & -1.994 \\
$\mathbf{3}$ & 100.2 & 192.0 & 55 & 300 & 511 & 3.42 & -5.430 & -2.010 \\
$\mathbf{4}$ & - & - & - & 300 & 507 & 3.45 & -5.404 & -1.954 \\
\hline
\end{tabular}

${ }^{a}$ 由 DSC/TGA 同步分析测定获得, 加热速率 $10{ }^{\circ} \mathrm{C} / \mathrm{min}, \mathrm{N}_{2}$ 保护 $;^{b}$ 在稀的 $\mathrm{CHCl}_{3}$ 溶液中测试; ${ }^{c}$ 激发波长: TPA, $364 \mathrm{~nm} ; \mathrm{DMTPA}, 370 \mathrm{~nm} ; m-\mathrm{CH}_{3}$-DMTPA, 373 $\mathrm{nm} ; p$ - $\mathrm{CH}_{3}$-DMTPA, $371 \mathrm{~nm} ; m-\mathrm{OCH}_{3}$-DMTPA, $375 \mathrm{~nm} ;{ }^{d}$ 由吸收开始点估算 $\left(E_{\mathrm{g}}=1240 / \lambda_{\text {onset }} \mathrm{eV}\right)^{[10]}$. 
$55.0 \%, R_{\mathrm{f}} 0.73$ [ $V$ (石油醚), $\left(60 \sim 90{ }^{\circ} \mathrm{C}\right): V(\mathrm{EtOAc})=$ $25: 1] .{ }^{1} \mathrm{H} \mathrm{NMR}\left(\mathrm{CDCl}_{3}, 500 \mathrm{MHz}\right) \delta: 7.22(\mathrm{t}, J=7.3 \mathrm{~Hz}$, 2H, ArH), 7.12 (td, $J=8.1,0.8 \mathrm{~Hz}, 1 \mathrm{H}, \operatorname{ArH}), 7.07$ (d, $J=$ $8.6 \mathrm{~Hz}, 2 \mathrm{H}, \mathrm{ArH}), 6.98$ (td, $J=7.3,1.0 \mathrm{~Hz}, 1 \mathrm{H}, \mathrm{ArH}), 6.72$ (s, 2H, ArH), $6.67(\mathrm{~s}, 1 \mathrm{H}, \mathrm{ArH}), 6.63(\mathrm{~d}, J=8.1 \mathrm{~Hz}, 1 \mathrm{H}$, ArH), 6.61 (dd, $J=3.7,2.1 \mathrm{~Hz}, 1 \mathrm{H}, \mathrm{ArH}), 6.53$ (ddd, $J=$ 8.2, 2.4, $1.1 \mathrm{~Hz}, 1 \mathrm{H}, \mathrm{ArH}), 3.70\left(\mathrm{~s}, 3 \mathrm{H}, \mathrm{OCH}_{3}\right), 2.21(\mathrm{~s}$, $6 \mathrm{H}, 2 \times \mathrm{CH}_{3}$ ); ${ }^{13} \mathrm{C}$ NMR (DMSO- $\left.d_{6}, 125 \mathrm{MHz}\right) \delta: 160.0$, $148.6,147.2,147.0,138.5,130.0,129.3,125.0,123.8$, 122.7, 122.1, 115.6, 109.1, 107.6, 54.9, 20.8; IR (film) v: 3032, 2916, 2860, 1590, 1490, 1470, 1456, 1378, 1318, $1204 \mathrm{~cm}^{-1}$. Anal. calcd for $\mathrm{C}_{21} \mathrm{H}_{21} \mathrm{~N}$ : C 83.13, H 6.98, N 4.62; found $\mathrm{C}$ 83.36, H 7.15, N 4.48. HRMS calcd for $\mathrm{C}_{21} \mathrm{H}_{21} \mathrm{NO}$ 303.1623, found 303.1616.

\section{3 性质测试}

利用 DSC/TGA 同步分析仪, 在扫描条件为 25 $600{ }^{\circ} \mathrm{C}$, 氮气保护, 升温速度为 $10{ }^{\circ} \mathrm{C} / \mathrm{min}$ 条件下, 考 察了 3 个固体目标化合物 DMTPA, $m$ - $\mathrm{CH}_{3}$-DMTPA, $p$ - $\mathrm{CH}_{3}$-DMTPA 的分解温度 $\left(T_{5 \mathrm{~d}}\right.$, 为失去 $5 \%$ 质量的温 度)、玻璃化转化温度 $\left(T_{\mathrm{g}}\right)$ 和熔点 $\left(T_{\mathrm{m}}\right)$. 利用 UV-vis 光谱 和荧光光谱研究了 4 个目标化合物在稀的 $\mathrm{CHCl}_{3}$ 溶液中 的光学性质; 利用循环伏安法 $(\mathrm{CV})$ 研究了目标化合物 的氧化还原性质, 样品的浓度为 $5 \times 10^{-4} \mathrm{~mol} / \mathrm{L}$ 的乙腈 溶液, 支持电解质为 $0.05 \mathrm{~mol} / \mathrm{L}$ 四丁基高氯酸胺(TBAP) 的乙腈溶液, 采用三电极体系, 工作电极为玻碳电极, 参比为饱和甘录电极, 对电极为铂丝电极, 扫描速率为 $50 \mathrm{mV} / \mathrm{s}$, 扫描电位 $0 \sim 1.6 \mathrm{~V}$, 扫描 3 次, 计算了 $\mathrm{HOMO}$ 和 LUMO 的能级. 将所测定数据与三苯胺(TPA, 空穴 传输材料)进行比较.

\section{2 结果与讨论}

\section{1 目标化合物的合成}

经典的 Ullmann 偶联反应合成方法是以铜粉或铜盐 作催化剂、金属氢氧化物或碳酸盐作碱, 硝基苯为溶剂, 由芳卤(常用芳碘)和芳胺进行反应. 但这种方法存在着 需要催化剂、高温、反应时间长、强碱、副反应多、产 率低、后处理困难等不足, 因此, 改进乌尔曼反应条件 的研究仍是目前研究的热点课题. 本文参考文献[8]报 道的合成方法, 成功地合成出了 4 个目标化合物, 除 DMPTA 之外，均为新化合物.

\section{2 目标化合物的谱图解析}

\subsubsection{IR 光谱}

目标化合物在 $3032 \sim 3036 \mathrm{~cm}^{-1}$ 处出现的中等强度 的吸收峰为苯环 $\mathrm{C}-\mathrm{H}$ 的伸缩振动吸收峰; 在 2916
$2856 \mathrm{~cm}^{-1}$ 处出现的吸收峰为饱和 $\mathrm{C}-\mathrm{H}$ 的反对称和对 称伸缩振动吸收峰; 在 $1590 \sim 1470 \mathrm{~cm}^{-1}$ 处出现的强吸 收峰为苯环骨架振动吸收峰; 在 $1380 \sim 1378 \mathrm{~cm}^{-1}$ 处出 现的强吸收峰为 $\mathrm{C}-\mathrm{H}$ 的弯曲振动吸收峰; 在 1318 $1300 \mathrm{~cm}^{-1}$ 处出现的强吸收峰为 $\mathrm{C}-\mathrm{N}$ 键的伸缩振动吸 收峰.

\subsubsection{NMR 谱}

目标化合物的 ${ }^{1} \mathrm{H}$ NMR 中, 在 $\delta 2.20 \sim 3.70$ 处出现 的单峰为 $\mathrm{CH}_{3}$ 或 $\mathrm{OCH}_{3}$ 中的质子吸收峰, 在 $\delta 6.53 \sim 7.22$ 处出现的峰为苯环上的质子吸收峰.

目标化合物的 ${ }^{13} \mathrm{C} N \mathrm{NMR}$ 中, 在 $\delta 107.6 \sim 160.0$ 处出 现的峰为芳环中碳的吸收峰, 在 $\delta 20.3 \sim 20.9$ 处出现的 峰为甲基碳的吸收峰, 在 $\delta 54.9$ 处出现的峰为甲氧基中 碳的吸收峰.

\section{3 热稳定性}

由表 1 结果表明, (1)供电子取代基的引入使 $T_{5 \mathrm{~d}}$ 提 高, 所合成的化合物的热稳定性较 TPA 高. (2)由 DSC 测 定可知, $T_{\mathrm{g}}$ (玻璃化转化温度)与 TPA 相同, 这说明所合 成的目标化合物形成分子玻璃及成膜能力与 TPA 相当, 有望应用于 OLEDs 之中.

\section{4 光学性质}

由表 1 可以看出, 目标化合物在 $298 \sim 300 \mathrm{~nm}$ 处出 现了一个强吸收峰, 这是 $\mathrm{N}-\mathrm{Ar}$ 的 $\pi-\pi$ *跃迁所致, 并且 由于 $\mathrm{CH}_{3}$ 的 $\sigma-\pi$ 超共轭和 $\mathrm{OCH}_{3}$ 的 $\mathrm{p}-\pi$ 共轭作用, 使其谱 带相对于 TPA 的谱带向红移动, 从而导致能隙 $\left(E_{\mathrm{g}}\right)$ 降低. 它们都是良好的发光体, 在可见光区发射波长为 502 $511 \mathrm{~nm}$, 像吸收光谱一样, $\mathrm{Ar}_{2} \mathrm{~N}$ 上对位基团对发射的影 响是敏感的, $\mathrm{Ar}_{2} \mathrm{~N}$ 上取代基的电子性质对发射波长的影 响顺序是: $p-\mathrm{CH}_{3}>m-\mathrm{OCH}_{3}>m-\mathrm{CH}_{3}>\mathrm{H}$. 这一事实证 明, 芳胺上取代基的位置将影响 HOMO 的性质, 供电子 基团能够提高 HOMO 能级.

\section{5 电化学性质}

为了改善有机感光器和 OLED 设备的性能(如更长 的寿命, 更高的效率等), 就必须要严格控制构成这些 设备的 HTMs 的性能和特性, 控制 HTMs 的 HOMO 能 级, 通过测定 HTMs 的氧化电位即可知其 HOMO 能 级 ${ }^{[7]}$. 本文利用循环伏安法 $(\mathrm{CV})$ 研究了目标化合物的氧 化还原性质, 计算了 HOMO 和 LUMO 的能级, 并将结 果与 TPA 相应的值进行了比较(表 1).

HOMO 和 LUMO 的能级可以根据氧化和还原的启 动电位计算得到. 测定有机化合物的 HOMO 能级数值 和 LUMO 数值一般通过先测定氧化电位 $E_{\mathrm{on}}^{\mathrm{OX}}$ 和带隙 $E_{\mathrm{g}}$, 再进行计算得到. 公式为(1)和(2) ${ }^{[11]}$ : 


$$
I_{\mathrm{p}}=E_{\mathrm{on}}^{\mathrm{OX}}+4.74
$$

$$
I_{\mathrm{p}}=E_{A}+E_{g}
$$

$I_{\mathrm{p}}$ 为离子化势, $E_{\mathrm{A}}$ 为电子亲和势. 氧化电位 $E_{\mathrm{on}}^{\mathrm{OX}}$ 通过循 环伏安法 ${ }^{[12]}$ 测得, 标准氢电极电位相对于真空能级为 $4.5 \mathrm{eV}$. 若选用饱和甘录电极作参比电极, 因为它相对 于标准氢电极电位为 $0.24 \mathrm{eV}$, 所以在公式中使用 4.74 . 又因为 $E_{\mathrm{HOMO}}=-\left|I_{\mathrm{p}}\right|, E_{\mathrm{LUMO}}=-\left|E_{\mathrm{A}}\right|$, 所以得到公式(3) 和(4) $)^{[13]}$.

$$
\begin{aligned}
& E_{\mathrm{HOMO}}=-\left|E_{\mathrm{on}}^{\mathrm{OX}}+4.74\right| \\
& E_{\mathrm{LUMO}}=E_{\mathrm{g}}+E_{\mathrm{HOMO}}
\end{aligned}
$$

由表 1 可见，在 TPA 上引入供电子的甲基和甲氧基 使得 $E_{\mathrm{HOMO}}$ 变大, $E_{\mathrm{HOMO}}$ 的增大可以提高空穴的注入效 率, 对于空穴的传输是有利的. 同时可以见到, 目标化 合物 $E_{\mathrm{LUMO}}$ 减小, $E_{\mathrm{LUMO}}$ 会使电子传输更加容易.

作为空穴传输材料, 还需要具有电化学稳定性. 因 此, 我们对 5 种化合物进行了多次的扫描, 发现它们均 具有良好的电化学稳定性. 仅以化合物 $m-\mathrm{OCH}_{3}-\mathrm{DMTP}$ 为例, 经过 3 次扫描之后, 曲线基本吻合, 说明多次的 氧化还原反应对其性能没有改变，电化学稳定性良好.

\section{References}

[1] Kurata, T.; Koshika, K.; Kato, F.; Kido, J.; Nishide, H. Polyhedron 2007, 26, 1776.

[2] Bellmann, E.; Shaheen, S. E.; Thayumanavan, S.; Barlow, S.; Grubbs, R. H.; Marder, S. R.; Kippelen, B.; Peyghambarian, N. Chem. Mater. 1998, 10, 1668.

[3] Kim, Y. K.; Hwang, S. H. Synth. Met. 2006, 156, 1028.

[4] Wang, Y.-G.; Ou, J.-B.; Xu, Q.-G.; Wu, L. Chin. J. Org. Chem. 2006, 26(7), 992 (in Chinese). (王延广, 欧加保, 徐强国, 吴琳, 有机化学, 2006, 26(7), 992.)

[5] Shirota, Y.J. Mater. Chem. 2005, 15, 75.

[6] Thelakkat, M. Macromol. Mater. Eng. 2002, 287, 442.

[7] Bender, T. P.; Graham, J. F.; Duff, J. M. Chem. Mater. 2001, 13, 4105.

[8] Goodbrand, H. B.; Hu, N. X. J. Org. Chem. 1999, 64, 670.

[9] J \& K Chemical LTD Acros Organics Reference Handbook of Fine Chemicals, Beijing, 2006 2007, p. 1845.

[10] Promarak, V.; Ichikawa, M.; Meunmart, D.; Sudyoadsuk, T.; Saengsuwan, S.; Keawina, T. Tetrahedron Lett. 2006, 47, 8949.

[11] Tan, C. X.; Feng, R. F.; Peng, X. X. Chin. Chem. Lett. 2007, 18, 505.

[12] Ding, B.-D.; Zhang, J.-M.; Zhu, W.-Q.; Zheng, X.-Y.; Wu, Y.-Z.; Jiang, X.-Y.; Zhang, Z.-L.; Xu, S.-H. Chin. J. Lumin. 2003, 24, 606 (in Chinese).

(丁邦东, 张积梅, 朱文清, 郑新友, 吴有智, 蒋雪茵, 张志林, 许少鸿, 发光学报, 2003, 24, 606.)

[13] Tian, W.-J.; Wu, F.; Fan, Y.-G.; Shen, J.-C. Chin. J. Lumin. 2000, 21,230 (in Chinese).

(田文晶, 吴芳, 樊玉国, 沈家骢, 发光学报, 2000, 21, 230.)

(Qin, X.; Lu, Z.) 